\title{
Sufficient Conditions on the Exponential Stability of Neutral Stochastic Differential Equations with Time-Varying Delays
}

\author{
Yanwei Tian $^{1}$ and Baofeng Chen ${ }^{2}$ \\ ${ }^{1}$ School of Mathematics and Statistics, Anyang Normal University, Anyang, Henan 455002, China \\ ${ }^{2}$ School of Mathematics and Physics, Anyang Institute of Technology, Anyang, Henan 455002, China \\ Correspondence should be addressed to Yanwei Tian; tyw994@126.com
}

Received 24 January 2014; Revised 26 April 2014; Accepted 26 April 2014; Published 12 May 2014

Academic Editor: Shuping He

Copyright (c) 2014 Y. Tian and B. Chen. This is an open access article distributed under the Creative Commons Attribution License, which permits unrestricted use, distribution, and reproduction in any medium, provided the original work is properly cited.

\begin{abstract}
The exponential stability is investigated for neutral stochastic differential equations with time-varying delays. Based on the Lyapunov stability theory and linear matrix inequalities (LMIs) technique, some delay-dependent criteria are established to guarantee the exponential stability in almost sure sense. Finally a numerical example is provided to illustrate the feasibility of the result.
\end{abstract}

\section{Introduction}

Neutral differential equations are well-known models from many areas of science and engineering, wherein, quite often the future state of such systems depends not only on the present state but also involves derivatives with delays. Deterministic neutral differential equations were originally introduced by Hale and Meyer [1] and discussed in Hale and Lunel (see [2]) and Kolmanovskii et al. (for details, see also $[3,4])$, among others. Motivated by chemical engineering systems as well as theory of aeroelasticity, stochastic neutral delay systems have been intensively studied over recent year [5-9]. Mao initiated the study of exponential stability of neutral stochastic differential delay equations in [5], while [9] incorporated Razumikhinis approach in neutral stochastic functional differential equations to investigate the stability problem. It is pointed out in Section 5 [10] that the conditions imposed in $[5,9]$ make the theory not applicable to the delay equation.

More recently, Luo et al. [6] proposed new criteria on exponential stability of neutral stochastic delay differential equations. In [11, 12], Milošević investigated the almost sure exponential stability of a class of highly nonlinear neutral stochastic differential equations with time-dependent delay, and some sufficient conditions were given for the considered systems. However, when the exponential stability of the neutral system with time-delay is considered, one always assumes that the derivative of the delay function is less than 1 (e.g., [6]). Meanwhile, the delay-independent conditions in $[6,10]$ are restricted when the delay is small. On the other hand, some results are proposed on stochastic Markovian jumping systems (e.g., [13-20]) and finite-time problems of stochastic systems (e.g., [18-22]), which can provide some useful methods and techniques for the neutral stochastic systems. This paper aims to develop the exponential stability in almost sure sense of the neutral stochastic differential equations with time-varying delays. Under the weaker assumptions that the derivative of time delay is less than some constant, sufficient conditions for the exponential stability are given in terms of linear matrix inequality (LMI) based on Lyapunov stability theory, which can be checked easily by MATLAB LMI Toolbox.

The paper is organized as follows. In the remainder of this section we recall some preliminaries, mainly from [5]. In Section 3 we state the main results on exponential stability. 
Section 4 will provide numerical examples to illustrate the feasibility and effectiveness of the results, and the conclusion will be made in Section 5 .

\section{Preliminaries}

Throughout this paper, unless otherwise specified, let $\{\Omega$, $\mathscr{F}, P\}$ be a complete probability space with a filtration $\left\{\mathscr{F}_{t}\right\}_{t \geq 0}$ satisfying the usual conditions (i.e., right continuous and $F_{0}$ containing all $P$-null sets). Let $w(t)=$ $\left(w_{1}(t), w_{2}(t), \ldots, w_{m}(t)\right)^{n}$ be $m$-dimensional Wiener process defined on the probability space. Let $|\cdot|$ denote the Euclidean norm in $\mathbb{R}^{n}$. $A^{T}$ stands for the transpose of the vector or matrix $A$. If $A$ is a matrix, its trace norm is denoted by $|A|=\sqrt{A^{T} A} \cdot a \vee b$ denotes $\max \{a, b\} . \lambda_{\max }(\cdot), \lambda_{\min }(\cdot)$ are maximum eigenvalue and minimum eigenvalue, respectively.

Consider the following $n$-dimensional neutral stochastic differential delay equations with time-varying delays:

$$
\begin{gathered}
\mathrm{d}[x(t)-G(x(t-\delta(t)))] \\
=f(x(t), x(t-\delta(t)), t) \mathrm{d} t \\
\quad+g(x(t), x(t-\delta(t)), t) \mathrm{d} w(t) \\
x(t)=\xi(t) \in C_{\mathscr{F}_{0}}^{b}\left([-\tau, 0], \mathbb{R}^{n}\right), \quad t \in[-\tau, 0],
\end{gathered}
$$

where $f: \mathbb{R}^{n} \times \mathbb{R}^{n} \times \mathbb{R}_{+} \rightarrow \mathbb{R}^{n}, g: \mathbb{R}^{n} \times \mathbb{R}^{n} \times$ $\mathbb{R}_{+} \rightarrow \mathbb{R}^{n \times m}$, and $G \in C\left(\mathbb{R}^{n}, \mathbb{R}^{n}\right)$. The functions $\delta(t):$ $\mathbb{R}_{+} \rightarrow[0, \tau]$ are continuously differentiable such that $0 \leq$ $\delta(t) \leq \delta, \dot{\delta}(t) \leq \bar{\delta}$. Let $C\left([-\tau, 0], \mathbb{R}^{n}\right)$ denote the family of continuous functions $\phi$ from $[-\tau, 0]$ to $\mathbb{R}^{n}$ with the norm $\|\phi\|=\sup _{-\tau \leq \theta \leq 0}|\phi(\theta)|$. Let $C_{\mathscr{F}_{0}}^{b}\left([-\tau, 0], \mathbb{R}^{n}\right)$ be the family of all $\mathscr{F}_{0}$-measurable $C\left([-\tau, 0], \mathbb{R}^{n}\right)$-valued random variables $\xi=\{\xi(\theta): \tau \leq \theta \leq 0\}$ such that $\sup _{-\tau \leq \theta \leq 0} E|\xi(\theta)|^{2}<\infty$. To guarantee the existence and uniqueness of the solution, we first list the following hypotheses.

$\left(\mathrm{H}_{1}\right)$ Both the functionals $f$ and $g$ satisfy the uniform Lipschitz conditions. That is, there is a diagonal positive matrix $L=\operatorname{diag}\left\{L_{1}, L_{2}, \ldots, L_{n}\right\}$ such that

$$
\begin{aligned}
|f(x, y, t)-f(\bar{x}, \bar{y}, t)| & \vee|g(x, y, t)-g(\bar{x}, \bar{y}, t)| \\
\leq & |L(x-\bar{x})|+|L(y-\bar{y})|
\end{aligned}
$$

for all $t \geq 0$ and those $x, y, \bar{x}, \bar{y} \in \mathbb{R}^{n}$.

$\left(\mathrm{H}_{2}\right)$ There is a constant $k \in(0,1)$ such that for all $\phi_{1}, \phi_{2} \in$ $C_{\mathscr{F}_{0}}^{b}\left([-\tau, 0], \mathbb{R}^{n}\right)$

$$
\left|G\left(\phi_{1}\right)-G\left(\phi_{2}\right)\right|^{2} \leq k \sup _{-\tau \leq \theta \leq 0}\left|\phi_{1}(\theta)-\phi_{2}(\theta)\right|^{2} .
$$

It is well known (see, e.g., [3]) that under hypotheses $\mathrm{H}_{1}, \mathrm{H}_{2}$ (1) has a unique continuous solution on $t \geq-\tau$.

To obtain sufficient conditions on almost sure exponential stability, the following lemmas and definition are given.

Lemma 1 (see [23]). For any positive definite constant matrix $M \in \mathbb{R}^{n \times n}$, scalar $r>0$, and vector function $f(\cdot):[0, r] \rightarrow$ $\mathbb{R}^{n}$ such that the integrations in the following are well defined, then the following inequality holds:

$$
\left(\int_{0}^{r} f(s) d s\right)^{T} M\left(\int_{0}^{r} f(s) d s\right) \leq r \int_{0}^{r} f^{T}(s) M f(s) d s .
$$

The following semimartingale convergence theorem will play an important role in the later parts.

Lemma 2 (see [24]). Let $A(t)$ and $U(t)$ be two continuous adapted increasing processed on $t \geq 0$ with $A(0)=U(0)=0$ a.s. Let $M(t)$ be a real-valued continuous local martingale with $M(0)=0$ a.s. Let $\varsigma$ be a nonnegative $\mathscr{F}_{0}$-measurable random variable. Define

$$
X(t)=\varsigma+A(t)-U(t)+M(t) \quad \text { for } t \geq 0 .
$$

If $X(t)$ is nonnegative, then

$$
\begin{aligned}
\left\{\lim _{t \rightarrow \infty} A(t)<\infty\right\} & \subset\left\{\lim _{t \rightarrow \infty} X(t)<\infty\right\} \\
& \cap\left\{\lim _{t \rightarrow \infty} U(t)<\infty\right\} \quad \text { a.s., }
\end{aligned}
$$

where $B \subset D$ a.s. means $P(B \cap D)=0$. In particular, if $\lim _{t \rightarrow \infty} A(t)<\infty$ a.s., then for almost all $w \in \Omega$,

$$
\lim _{t \rightarrow \infty} X(t)<\infty, \quad \lim _{t \rightarrow \infty} U(t)<\infty
$$

that is, both $X(t)$ and $U(t)$ converge to finite random variables.

Definition 3 (see [25]). The equilibrium of solution $\{x(t), t \geq$ 0 \} of (1) is said to be almost sure exponentially stable if there exists a constant $\varepsilon>0$ such that

$$
\limsup _{t \rightarrow \infty} \frac{1}{t} \log |x(t)| \leq-\varepsilon \quad \text { a.s. }
$$

for any bounded initial condition $\xi$.

\section{Main Results}

Theorem 4. Let hypotheses $H_{1}, H_{2}$ hold. System (1) is almost sure exponentially stable, if there exists positive definite matrix such that the following LMI holds 


$$
\Omega=\left(\begin{array}{cccccc}
\Xi_{11} & N_{2}^{T}-N_{1}^{T} & N_{3}^{T}+U^{T} & N_{4}^{T} & -N_{1}^{T}+N_{5}^{T} & 0 \\
* & \Xi_{22} & -N_{3}^{T} & -N_{4}^{T} & -N_{2}^{T}-N_{5}^{T} & 0 \\
* & * & \Xi_{33} & 0 & -N_{3}^{T} & U \\
* & * & * & \Xi_{44} & -N_{4}^{T} & 0 \\
* & * & * & * & -\frac{1}{\delta} R-2 N_{5}^{T} & 0 \\
* & * & * & * & * & -\varepsilon I
\end{array}\right)<0,
$$

where

$$
\begin{aligned}
\Xi_{11}= & \beta P+e^{\beta \delta} \mathrm{Q}+P+2 N_{1}^{T}, \\
\Xi_{22}= & \left(-(1-\bar{\delta}) e^{\beta \delta} \vee-(1-\bar{\delta})\right) Q-2 N_{2}, \\
\Xi_{33}= & -2 U+e^{\beta \delta} S+2 \lambda_{\max }(P) L^{T} L \\
& +\frac{2}{\beta} \lambda_{\max }(R)\left(e^{\beta \delta}-1\right) L_{1}, \\
\Xi_{44}= & -(1-\bar{\delta}) S+2 \lambda_{\max }(P) L^{T} L \\
& +\frac{2}{\beta} \lambda_{\max }(R)\left(e^{\beta \delta}-1\right) L_{1}+\varepsilon k I .
\end{aligned}
$$

Proof. To confirm that the stochastic neutral differential equation (1) is mean-square exponentially stable with decay rate $\beta$, we define a Lyapunov-Krasovskii functional $V(x(t), t)$ as follows:

$$
\begin{aligned}
V(x(t), t)= & e^{\beta t} \rho^{T}(t) P \rho(t) \\
& +\int_{t-\delta(t)}^{t} e^{\beta(s+\delta)} \rho^{T}(s) Q \rho(s) \mathrm{d} s \\
& +\int_{-\delta}^{0} \int_{t+\theta}^{t} e^{\beta(s-\theta)} f^{T}(x(s), y(s), s) \\
& +\int_{t-\delta(t)}^{t} e^{\beta(s+\delta)} x^{T}(s) S x(s) \mathrm{d} s .
\end{aligned}
$$

For simplicity, let $y(t)=x(t-\delta(t)), \rho(t)=x(t)-G(y(t))$. By generalizing Ito's formula, we have that

$$
E V(\rho(t), t)=E V(\rho(0), 0)+\int_{0}^{t} \mathscr{L} V(x(s), y(s), s) \mathrm{d} s
$$

Then, the derivative of $V(\rho(t), t)$ along the solution of (1) gives

$$
\begin{aligned}
L V( & x(t), y(t), t) \\
= & \beta e^{\beta t} \rho^{T}(t) P \rho(t)+2 e^{\beta t} \rho(t)^{T} P f(x(t), y(t), t) \\
& +e^{\beta t} g^{T}(x(t), y(t), t) P g(x(t), y(t), t) \\
& +e^{\beta(t+\delta)} \rho(t) Q \rho(t) \\
& -e^{\beta(t-\delta(t))+\delta} \rho^{T}(t-\delta(t)) Q \rho(t-\delta(t))(1-\dot{\delta}(t)) \\
& +\frac{1}{\beta} e^{\beta t}\left(e^{\beta \delta}-1\right) f^{T}(x(t), y(t), t) R f(x(t), y(t), t) \\
& -e^{\beta t} \int_{t-\delta}^{t} f^{T}(x(s), y(s), s) R f(x(s), y(s), s) \mathrm{d} s \\
& +e^{\beta(t+\delta)} x(t) S x(t)-e^{\beta(t-\delta(t))+\delta} \\
& \times x^{T}(t-\delta(t)) S x(t-\delta(t))(1-\dot{\delta}(t)) .
\end{aligned}
$$

Note that, from system (1) and Newton-Leibniz formula, we have

$$
\begin{aligned}
M= & \left(\rho(t)-\rho(t-\delta(t))-\int_{t-\delta(t)}^{t} f(x(s), y(s), s) \mathrm{d} s\right. \\
& \left.-\int_{t-\delta(t)}^{t} g(x(s), y(s), s) \mathrm{d} w(s)\right)=0 .
\end{aligned}
$$

By calculation, it is clear that

$$
\begin{aligned}
f^{T}(x & (t), y(t), t) R f(x(t), y(t), t) \\
& \leq \lambda_{\max }(R) f^{T}(x(t), y(t), t) f(x(t), y(t), t) \\
& \leq \lambda_{\max }(R)|f(x(t), y(t), t)|^{2} \\
& \leq 2 \lambda_{\max }(R)\left(x^{T}(t) L^{T} L x(t)+x^{T}(t-\delta(t)) L^{T} L y(t)\right),
\end{aligned}
$$


and then by which, we have

$$
\begin{aligned}
& 2 \rho^{T}(t) P f(x(t), y(t), t) \\
& \leq \rho^{T}(t) P^{T} \rho(t) \\
&+f^{T}(x(t), y(t), t) P f(x(t), y(t), t) \\
& \leq \rho^{T}(t) P^{T} \rho(t) \\
&+2 \lambda_{\max }(P)\left(x^{T}(t) L^{T} L x(t)+y^{T}(t) L^{T} L y(t)\right), \\
&-e^{\beta(t-\delta(t))+\delta} \rho^{T}(t) Q \rho(t)(1-\dot{\delta}(t)) \leq-(1-\bar{\delta}) e^{\beta t} \rho^{T}(t) Q \rho(t) . \\
&-e^{\beta(t-\delta(t))+\delta} x^{T}(t) S x(t)(1-\dot{\delta}(t)) \\
& \leq-(1-\bar{\delta}) e^{\beta t} x^{T}(t) S x(t) .
\end{aligned}
$$

Moreover, by Lemma 2, one can get

$$
\begin{aligned}
& -\int_{t-\delta}^{t} f^{T}(x(s), y(s), s) R f(x(s), y(s), s) \mathrm{d} s \\
& \leq-\int_{t-\delta(t)}^{t} f^{T}(x(s), y(s), s) R f(x(s), y(s), s) \mathrm{d} s \\
& \leq-\frac{1}{\delta}\left(\int_{t-\delta(t)}^{t} f(x(s), y(s), s) \mathrm{d} s\right)^{T} \\
& \quad \times R\left(\int_{t-\delta(t)}^{t} f(x(s), y(s), s) \mathrm{d} s\right) .
\end{aligned}
$$

Letting $L_{1}=L^{T} L$ and substituting (14)-(17) into (13) yield

$$
\begin{aligned}
& L V(x(t), y(t), t) \\
& \leq e^{\beta t}\left\{\beta \rho^{T}(t) P \rho(t)+\rho^{T}(t) P^{T} \rho(t)\right. \\
&+2 \lambda_{\max }(P) x^{T}(t) L_{1} x(t) \\
&+2 \lambda_{\max }(P) y^{T}(t) L_{1} y(t) \\
&+\left(-(1-\bar{\delta}) e^{\beta \delta} \vee-(1-\bar{\delta})\right) \rho^{T}(t) Q \rho(t) \\
&+e^{\beta \delta} \rho^{T}(t) Q_{1} \rho(t)+2 \lambda_{\max } \frac{1}{\beta}\left(e^{\beta \delta}-1\right) \\
& \times\left(x^{T}(t) L_{1} x(t)+y^{T}(t) L_{1} y(t)\right) \\
&-\frac{1}{\delta}\left(\int_{t-\delta(t)}^{t} f(x(s), y(s), s) \mathrm{d} s\right)^{T} \\
&\left.\times R\left(\int_{t-\delta(t)}^{t} f(x(s), y(s), s) \mathrm{d} s\right)\right\} .
\end{aligned}
$$

Furthermore, from (14), it follows that

$$
\begin{aligned}
& A=2 \eta^{T}\left(N_{1}^{T}, N_{2}^{T}, N_{3}^{T}, N_{4}^{T}, N_{5}^{T}\right)^{T} M=0, \\
& B=2 x^{T}(t) U[\rho(t)-x(t)+G(y(t))]=0,
\end{aligned}
$$

where $\eta=\left(\rho^{T}, \rho^{T}(t-\delta(t)), x^{T}(t), y^{T}(t),\left(\int_{t-\delta(t)}^{t} f(x, y, s)\right.\right.$ $\left.\mathrm{d} s)^{T}\right)^{T}$, and $N_{i}(1 \leq i \leq 5), U$ are matrices with compatible dimensions.

$$
\text { It can be shown that }
$$

$$
\begin{aligned}
& \int_{0}^{t} \mathscr{L} V(x(s), y(s), s) \mathrm{d} s \\
& \quad+e^{\beta t}(A+B)+e^{\beta t} M^{T} P_{1}\left(M(t)+\int_{t-\delta(t)}^{t} g(x, y, s) \mathrm{d} s\right)
\end{aligned}
$$$$
\leq e^{\beta t}\left\{\beta \rho^{T}(t) P \rho(t)+\rho^{T}(t) P^{T} \rho(t)\right.
$$

$$
\begin{aligned}
&+x^{T}(t) L_{1} x(t)+e^{\beta \delta} \rho^{T}(t) Q \rho(t) \\
&+ y^{T}(t) L_{1} y(t)+2 \lambda_{\max }(P) x^{T}(t) L_{1} x(t) \\
&+ 2 \lambda_{\max } y^{T}(t) L_{1} y(t) \\
&-(1-\bar{\delta}) \rho^{T}(t) Q \rho(t)+e^{\beta \delta} x^{T}(t) S x(t) \\
&-(1-\bar{\delta}) x^{T}(t) S x(t)+2 \lambda_{\max } \frac{1}{\beta}\left(e^{\beta \delta}-1\right) \\
& \times\left(x^{T}(t) L_{1} x(t)+y^{T}(t) L_{1} y(t)\right) \\
&-\frac{1}{\delta}\left(\int_{t-\delta(t)}^{t} f(x(s), y(s), s) \mathrm{d} s\right)^{T} \\
&\left.\times R\left(\int_{t-\delta(t)}^{t} f(x(s), y(s), s) \mathrm{d} s\right)\right\} \\
& \leq e^{\beta t}\left\{\eta^{T} \widetilde{\Omega} \eta+\varepsilon^{-1} x^{T}(t) U U^{T} x(t)\right\}
\end{aligned}
$$

where $\Omega$ is defined as

$$
\widetilde{\Omega}=\left(\begin{array}{ccccc}
\Xi_{11} & N_{2}^{T}-N_{1}^{T} & N_{3}^{T}+U^{T} & N_{4}^{T} & -N_{1}^{T}+N_{5}^{T} \\
* & \Xi_{22} & -N_{3}^{T} & -N_{4}^{T} & -N_{2}^{T}-N_{5}^{T} \\
* & * & \Xi_{33} & 0 & -N_{3}^{T} \\
* & * & * & \Xi_{44} & -N_{4}^{T} \\
* & * & * & * & -\frac{1}{\delta} R-2 N_{5}^{T}
\end{array}\right) .
$$


By Schur complement, we know that $\eta^{T} \widetilde{\Omega} \eta+$ $\varepsilon^{-1} x^{T}(t) U U^{T} x(t)<0$. On the other hand, it follows that

$$
\begin{aligned}
V(\rho(t), t)= & V(\rho(0), 0)+\int_{0}^{t} \mathscr{L} V(x(s), y(s), s) \mathrm{d} s \\
& +\int_{0}^{t} 2 e^{\beta s} x^{s}(t) g(x(s), y(s), s) \mathrm{d} w(s) .
\end{aligned}
$$

Note that $\xi$ is bounded and $V, G$ are continuous; then $V(\rho(0))$ must be nonnegative bounded. Moreover, $\mathscr{L} V(x, y, t) \leq 0$ can be obtained directly:

$$
\begin{aligned}
V(\rho(t), t) \leq & V(\rho(0), 0) \\
& +\int_{0}^{t} 2 e^{\beta t} x^{T}(s) g(x(s), y(s), s) \mathrm{d} w(s) .
\end{aligned}
$$

By applying Lemma 2 to (23), one sees that

$$
\lim _{t \rightarrow \infty} \sup V(\rho(t), t)<\infty ;
$$

hence there exists a positive random variable $\zeta$ satisfying

$$
\sup _{0 \leq t<\infty} e^{\beta t}|x(t)-G(y(t))|^{2} \leq \zeta .
$$

Since, for any $\varepsilon_{3} \in(0,1)$

$$
\begin{aligned}
\mid x(t) & -\left.G(y(t))\right|^{2} \\
& \geq\left(1-\varepsilon_{3}^{-1}\right)|x(t)|^{2}-\left(\varepsilon_{3}-1\right)|G(y(t))|^{2},
\end{aligned}
$$

we must have

$$
\begin{aligned}
\sup _{0 \leq t \leq T} e^{\beta t}|x(t)|^{2} & \leq \zeta+\frac{k^{2}}{\varepsilon_{3}} \sup _{0 \leq t \leq T} e^{\beta t}|y(t)|^{2} \\
& \leq \zeta+k^{2} e^{\beta \tau}\|\xi\|^{2}+\frac{k^{2}}{\varepsilon_{3}} e^{\beta \tau} \sup _{0 \leq t \leq T} e^{\beta t}|x(t)|^{2} .
\end{aligned}
$$

From the above inequality (26), it yields the desired result

$$
\limsup _{t \rightarrow \infty} \frac{1}{t} \log |x(t)| \leq-\frac{\beta}{2} .
$$

That completes the proof.

\section{Example}

In this section, a numerical example will be given to illustrate that the proposed method is effective.

Example 1. Consider the following system:

$$
\begin{aligned}
d\left[x_{1}(t)-0.1 x_{2}(t-\delta(t))\right]= & -x_{1}(t) x_{2}(t-\delta(t)) \mathrm{d} t \\
& +x_{1}(t) \sin ^{2}(t-\delta(t)) \mathrm{d} \omega(t), \\
d\left[x_{2}(t)-0.1 x_{1}(t-\delta(t))\right]= & -x_{2}(t) x_{1}(t-\delta(t)) \mathrm{d} t \\
& +x_{2}(t) \cos ^{2}(t-\delta(t)) \mathrm{d} \omega(t),
\end{aligned}
$$

where the delay function is defined as $\delta(t)=(1 / 4) \sin (t), t>$ 0 . It is obvious that (29) satisfies the assumptions $\mathrm{H}_{1}$ and $\mathrm{H}_{2}$, and here $L=I, k=0.1$. Moreover, since $\dot{\delta}=(1 / 4) \cos (t)$, then $\bar{\delta}=1 / 4$.

According to Theorem 4 and employing MATLAB LMI Toolbox, it is relatively easy to deduce that the neutral stochastic differential equation (29) is almost sure exponentially stable.

Remark 5. Comparing with some existing sufficient criteria for neural stochastic differential equations (e.g., $[6,11,12]$ ), the obtained result is given in terms of linear matrix inequality (LMI), which can be easily checked by MATLAB LMI Toolbox.

\section{Conclusion}

The exponential stability is investigated for a class of neutral stochastic differential equations with time-varying delays. In order to overcome the difficulties, we introduce suitable Lyapunov functionals and employ linear matrix inequalities (LMIs) technique, and then a delay-dependent criteria are given to check the almost sure exponential stability of the concerned equations.

\section{Conflict of Interests}

The authors declare that there is no conflict of interests regarding the publication of this paper.

\section{References}

[1] J. K. Hale and K. R. Meyer, A Class of Functional Equations of Neutral Type, Memoirs of the American Mathematical Society, no. 76, American Mathematical Society, Providence, RI, USA, 1967.

[2] J. K. Hale and S. M. V. Lunel, Introduction to Functional Differential Equations, Springer, Berlin, Germany, 1991.

[3] V. B. Kolmanovskii and V. R. Nosov, Stability of FunctionalDifferential Equations, vol. 180 of Mathematics in Science and Engineering, Academic Press, New York, NY, USA, 1986.

[4] V. B. Kolmanovskii and A. Myshkis, Applied Theory of Functional Differential Equations, Kluwer Academic Publishers, Norwell, Mass, USA, 1992.

[5] X. Mao, "Exponential stability in mean square of neutral stochastic differential-functional equations," Systems \& Control Letters, vol. 26, no. 4, pp. 245-251, 1995.

[6] Q. Luo, X. Mao, and Y. Shen, "New criteria on exponential stability of neutral stochastic differential delay equations," Systems \& Control Letters, vol. 55, no. 10, pp. 826-834, 2006.

[7] V. B. Kolmanovskii and A. Myshkis, Introduction to the Theory and Applications of Functional Differential Equations, Kluwer Academic Publishers, Dordrecht, The Netherlands, 1999.

[8] M. S. Mahmoud, "Robust $H_{\infty}$ control of linear neutral systems," Automatica, vol. 36, no. 5, pp. 757-764, 2000.

[9] X. Mao, "Razumikhin-type theorems on exponential stability of neutral stochastic functional-differential equations," SIAM Journal on Mathematical Analysis, vol. 28, no. 2, pp. 389-401, 1997. 
[10] X. Mao, "Asymptotic properties of neutral stochastic differential delay equations," Stochastics and Stochastics Reports, vol. 68, no. 3-4, pp. 273-295, 2000.

[11] M. Milošević, "Highly nonlinear neutral stochastic differential equations with time-dependent delay and the Euler-Maruyama method," Mathematical and Computer Modelling, vol. 54, no. 910, pp. 2235-2251, 2011.

[12] M. Milošević, "Almost sure exponential stability of solutions to highly nonlinear neutral stochastic differential equations with time-dependent delay and the Euler-Maruyama approximation," Mathematical and Computer Modelling, vol. 57, no. 3-4, pp. 887-899, 2013.

[13] S. He, "Resilient $L_{2}-L_{\infty}$ filtering of uncertain Markovian jumping systems within the finite-time interval," Abstract and Applied Analysis, vol. 2013, Article ID 791296, 7 pages, 2013.

[14] S. He and F. Liu, "Adaptive observer-based fault estimation for stochastic Markovian jumping systems," Abstract and Applied Analysis, vol. 2012, Article ID 176419, 11 pages, 2012.

[15] Z. Wu, P. Shi, H. Su, and J. Chu, "Asynchronous $L_{2}-L_{\infty}$ filtering for discrete-time stochastic Markov jump systems with randomly occurred sensor nonlinearities," Automatica, vol. 50, no. 1, pp. 180-186, 2014.

[16] H. Shen, Y. Chu, S. Xu, and Z. Zhang, "Delay-dependent $H_{\infty}$ control for jumping delayed systems with two markov processes," International Journal of Control, Automation and Systems, vol. 9, no. 3, pp. 437-441, 2011.

[17] H. Shen, J. Park, L. Zhang, and Z. Wu, "Robust extended dissipative control for sampled-data Markov jump systems," International Journal of Control, 2014.

[18] J. Cheng, H. Zhu, S. Zhong, Y. Zhang, and Y. Li, "Finite-time $H_{\infty}$ control for a class of discrete-time Markov jump systems with partly unknown time-varying transition probabilities subject to average dwell time switching," Journal of Systems Science, 2013.

[19] J. Cheng, H. Zhu, S. Zhong, Y. Zeng, and L. Hou, "Finitetime $H_{\infty}$ filtering for a class of discrete-time Markovian jump systems with partly unknown transition probabilities," International Journal of Adaptive Control and Signal Processing, 2013.

[20] J. Cheng, H. Zhu, S. Zhong, Y. Zeng, and X. Dong, "Finite-time $H_{\infty}$ control for a class of Markovian jump systems with modedependent time-varying delays via new Lyapunov functionals," ISA Transactions, vol. 52, pp. 768-774, 2013.

[21] S. He and F. Liu, "Finite-time $H_{\infty}$ control of nonlinear jump systems with timedelays via dynamic observer-based state feedback," IEEE Transactions on Fuzzy Systems, vol. 20, no. 4, pp. 605-614, 2012.

[22] S.-P. He and F. Liu, "Robust finite-time $H_{\infty}$ control of stochastic jump systems," International Journal of Control, Automation and Systems, vol. 8, no. 6, pp. 1336-1341, 2010.

[23] R. Sh. Liptser and A. N. Shiryayev, Theory of Martingales, vol. 49 of Mathematics and Its Applications (Soviet Series), Kluwer Academic Publishers, Dordrecht, The Netherlands, 1989.

[24] K. Gu, "An integral inequality in the stability problem of time-delay systems," in Proceedings of the 39th IEEE Confernce on Decision and Control, pp. 2805-2810, Sydney, Australia, December 2000.

[25] Q. Luo and Y. Zhang, "Almost sure exponential stability of stochastic reaction diffusion systems," Nonlinear Analysis: Theory, Methods \& Applications, vol. 71, no. 12, pp. e487-e493, 2009. 


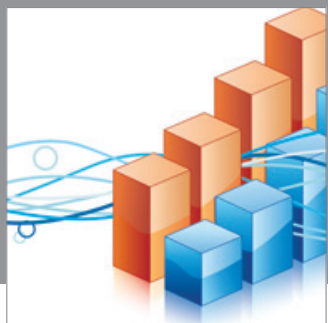

Advances in

Operations Research

mansans

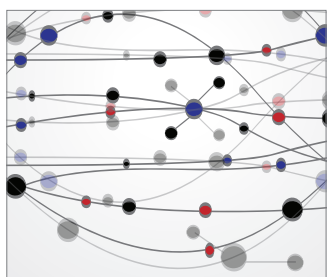

The Scientific World Journal
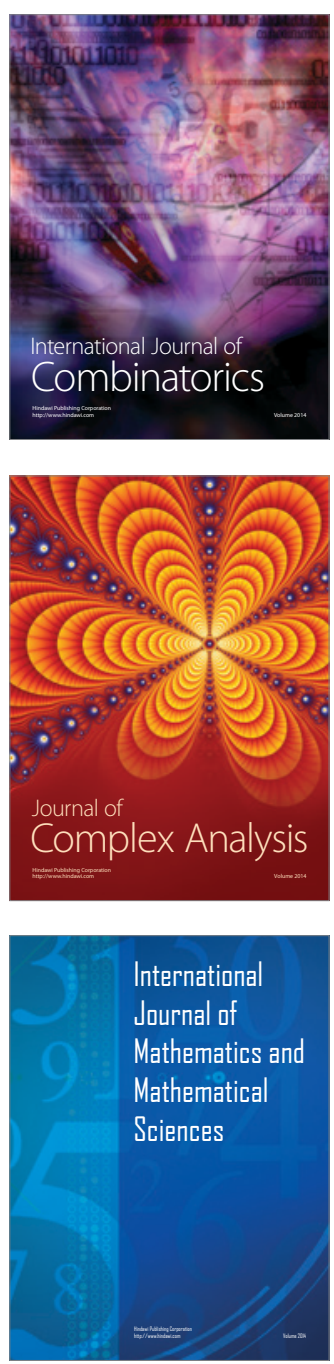
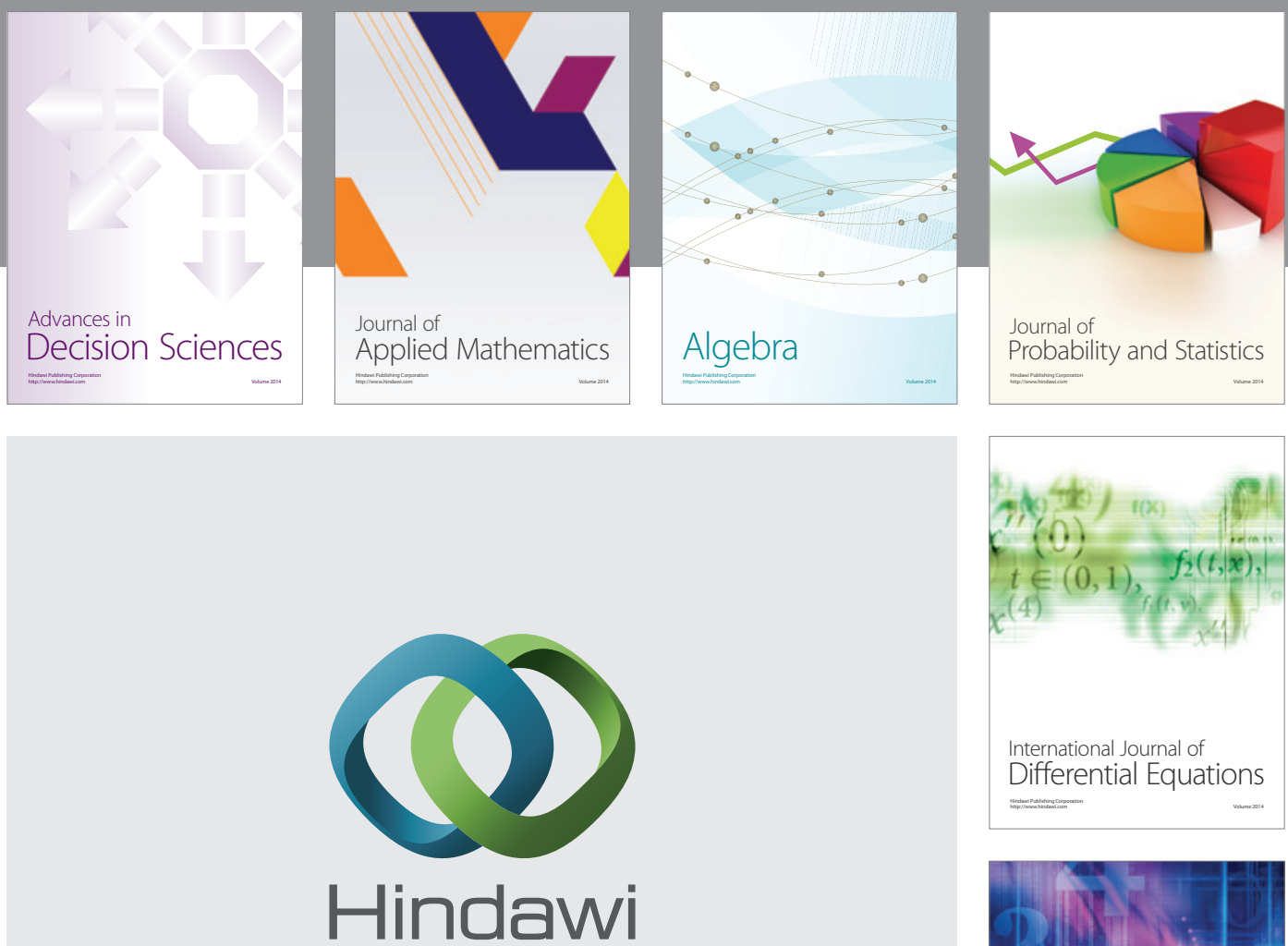

Submit your manuscripts at http://www.hindawi.com
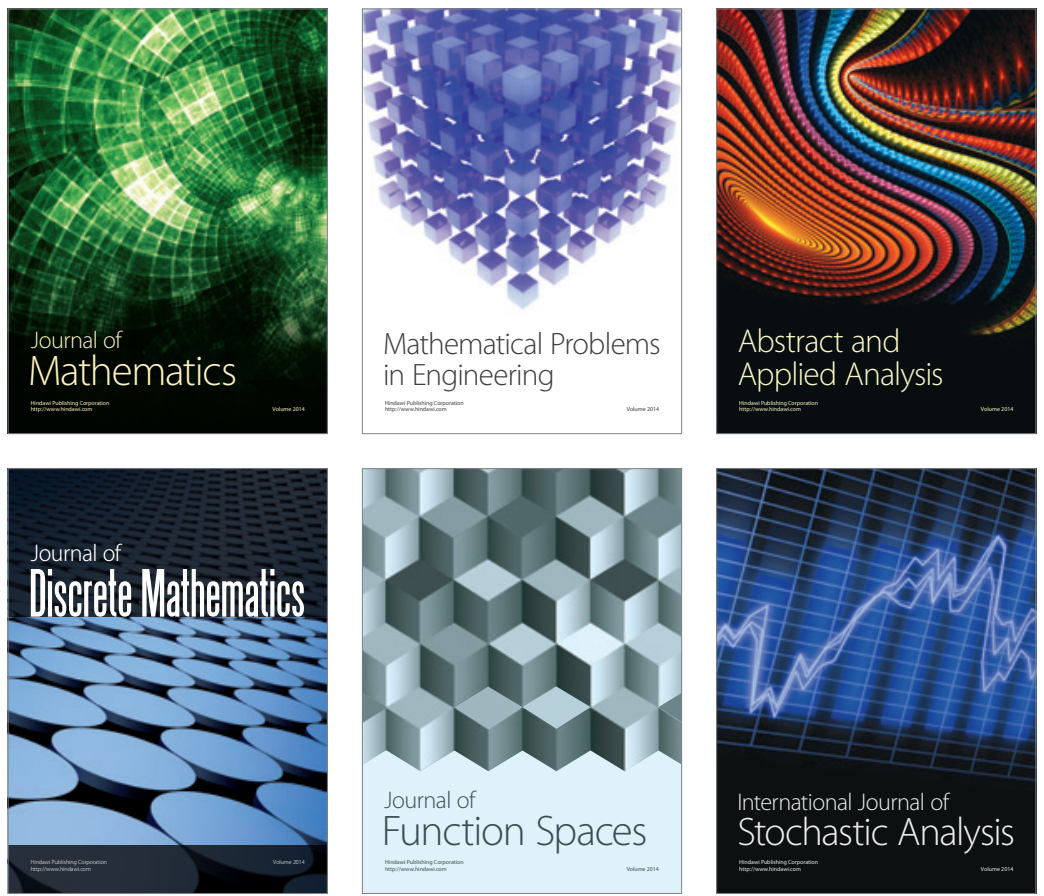

Journal of

Function Spaces

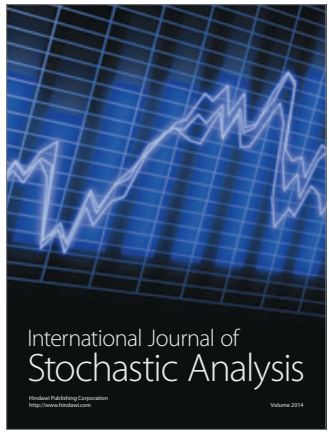

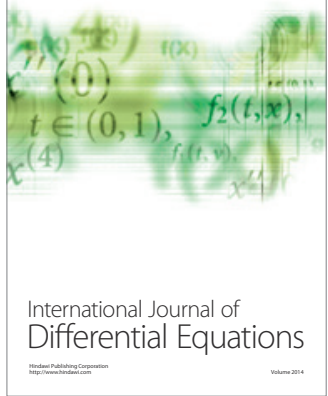
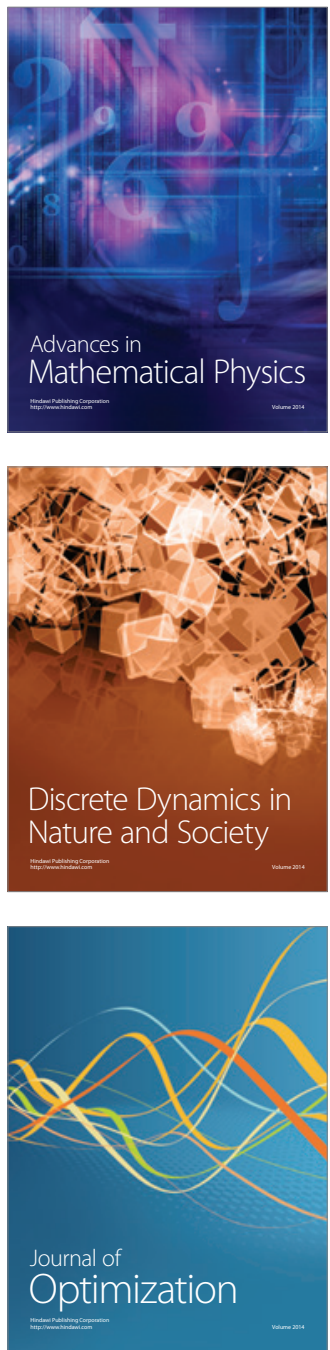